\title{
Hohe Erwartungen an Unternehmen
}

\section{Ergebnisse einer Befragung der Schweizer Bevölkerung zur verantwortungsvollen Unternehmensführung}

\author{
Bettina Furrer · Thea Weiss Sampietro
}

Online veröffentlicht: 8.3.2007

(c) Springer-Verlag 2007

Die gesellschaftliche Verantwortung von Unternehmen, ihre Corporate Social Responsibility (CSR) (1), hat in den letzten Jahren nicht zuletzt vor dem Hintergrund zahlreicher Firmenskandale eine starke Beachtung und Verbreitung erfahren (Dawkins und Lewis 2003:185). Erwartungen gegenüber Unternehmen, verantwortungsbewusst zu handeln, sind nicht neu (Carroll 1999:268). Von Unternehmen wird zunehmend erwartet, dass sie ökologische, soziale und ethische Standards einhalten, die über gesetzliche Anforderungen hinausgehen (Environics International 2003:15).

Der „Swiss CSR Monitor 2006“ (siehe Abbildung 1) hat zum Ziel, die öffentliche Meinung in der Schweiz zur gesellschaftlichen Verantwortung von Unternehmen zu erheben und Unternehmen damit Hinweise zu liefern, wo ökologische und soziale Chancen und Risiken ihrer Tätigkeiten liegen.

\section{Klare Erwartungen an Unternehmen}

$72 \%$ der Schweizer Bevölkerung erwarten von Unternehmen mehr als das Erwirtschaften von Gewinnen und die Einhaltung von Gesetzen. Vielmehr sollen Unternehmen auch zu einer besseren Gesellschaft beitragen. Dabei stehen für die Befragten die Ausbildung (36\% der Befragten), die

Bettina Furrer (dipl. natw. ETH), Dozentin

Thea Weiss Sampietro (lic. phil. I), wissenschaftliche Mitarbeiterin Zürcher Hochschule Winterthur

Institut für Nachhaltige Entwicklung

Postfach 805, 8401 Winterthur, Schweiz

Tel.: +4152 2677669 (dir.)

Fax.: +41 522687669 (dir.)

E-Mail: bettina.furrer@zhwin.ch

Info: www.ine.zhwin.ch
Der „Swiss CSR Monitor“ ist Teil einer internationalen Befragung von insgesamt über 20000 Personen zum Thema Unternehmensverantwortung, die unter der Leitung des internationalen Marktforschungsinstitutes GlobeScan seit sieben Jahren von verschiedenen Instituten in jeweils rund 20 Ländern durchgeführt wird.

Für den „Swiss CSR Monitor 2006“ wurden insgesamt 800 repräsentativ ausgewählte Personen über 18 Jahren in der deutsch- und französischsprachigen Schweiz telefonisch befragt. Die Daten wurden vom Institut für Angewandte Medienwissenschaft der Zürcher Hochschule Winterthur (IAM-ZHW) erhoben.

Die detaillierte Studie des Instituts für Nachhaltige Entwicklung der Zürcher Hochschule Winterthur (INEZHW) entstand in Zusammenarbeit mit dem CCRS (Centre for Corporate Responsibility and Sustainability) an der Universität Zürich.

Abb. 1 Details zum „Swiss CSR Monitor“

Armutsbekämpfung (17\%) und der Umweltschutz (14\%) im Vordergrund. Die Glaubwürdigkeit eines Unternehmens bezüglich CSR zeigt sich für die Schweizer Bevölkerung in der Zusammenarbeit von Firmen mit Nicht-Regierungsorganisationen (NGOs) (28\% der Befragten), dem Anbringen von Hinweisen und Labeln auf Produkten $(27 \%)$ und der Publikation eines CSR-Berichtes (21\%). $70 \%$ der Befragten denken allerdings, dass Unternehmen in erster Linie sozial verantwortlich handeln, um ihr Image zu verbessern und nicht um positive Beiträge zur Gesellschaft zu leisten. Ein Drittel der Befragten ist der Meinung, Firmen sollten gesetzlich zu verantwortungsvoller Unternehmensführung verpflichtet werden. 
Abb. 2 Sanktionsmassnahmen gegen verantwortungslos handelnde Unternehmen
Falls eine Firma nicht verantwortungsvoll handelt, würden Sie...

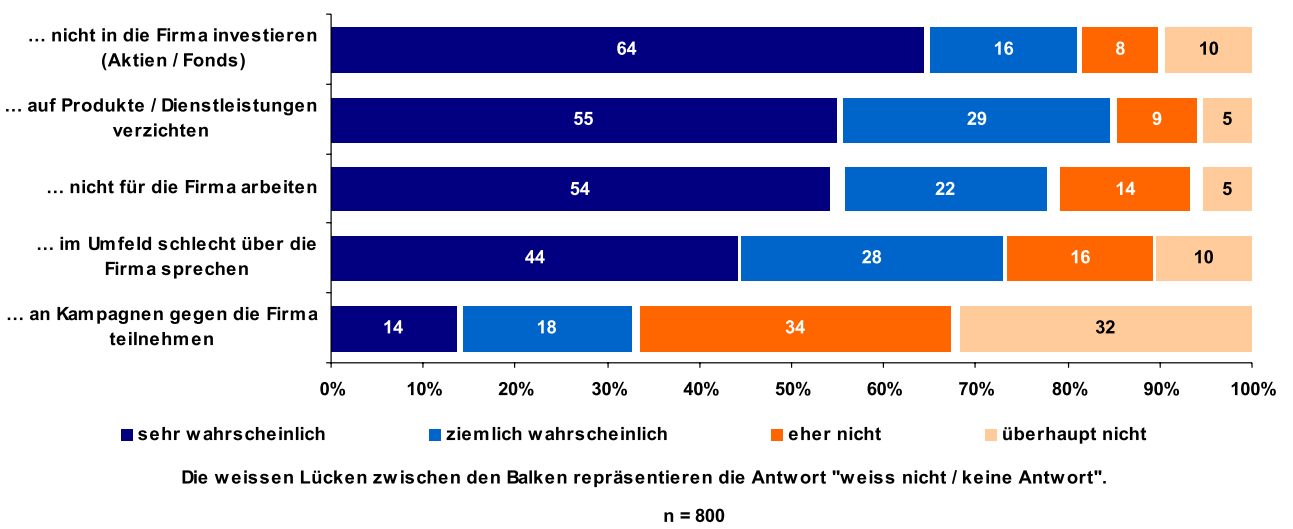

Aus welchem Grund schätzen Sie das Unternehmen als besonders verantwortungsbewusst ein?

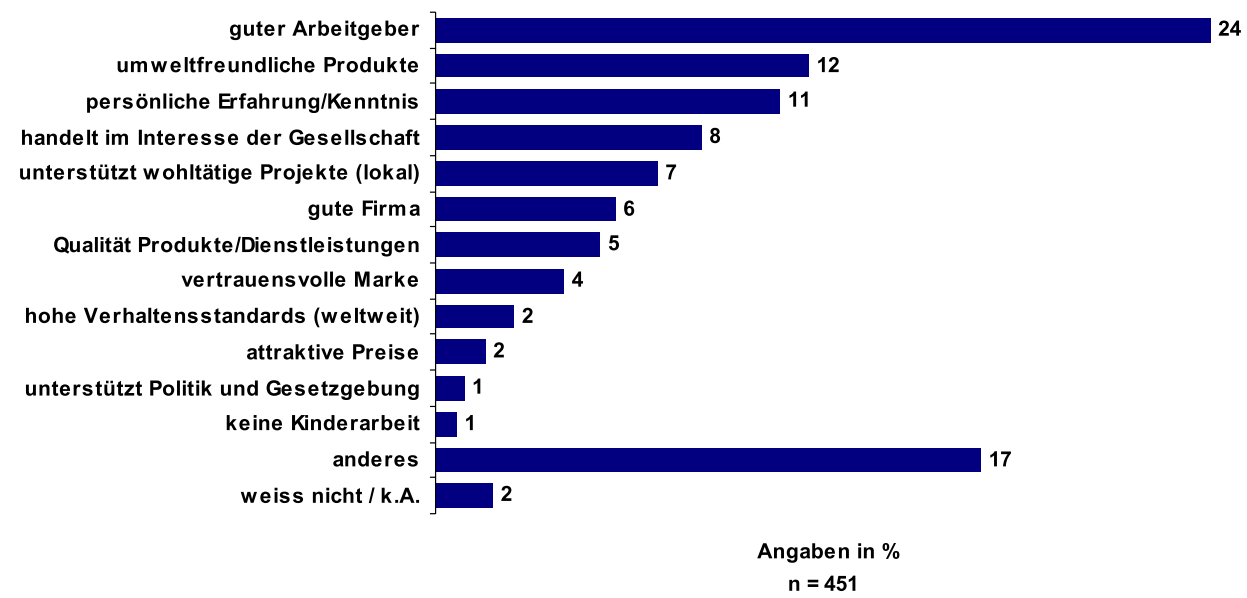

Abb. 3 Gründe für die positive Beurteilung von Firmen

\section{Bereitschaft zur Sanktionierung}

Bei der Schweizer Bevölkerung sind die Erwartungen an Unternehmen im Vergleich mit G8-Ländern hoch. Insbesondere haben die Schweizerinnen und Schweizer im internationalen Vergleich eine hohe Bereitschaft, Unternehmen zu sanktionieren, welche in ihrer Wahrnehmung verantwortungslos handeln. $64 \%$ der Befragten würden sehr wahrscheinlich nicht in ein solches Unternehmens investieren, $55 \%$ auf deren Produkte und Dienstleistungen verzichten und $54 \%$ nicht für eine solche Firma arbeiten (siehe Abbildung 2). Relativiert werden diese Aussagen allerdings dadurch, dass nur je rund die Hälfte der Befragten ein verantwortungsvoll resp. verantwortungslos handelndes Unternehmen nennen kann.

\section{Unternehmen kommunizieren zurückhaltend}

Die Befragung zeigt, dass die CSR-Anstrengungen der Unternehmen kaum wahrgenommen werden. Nur $35 \%$ der Be- fragten haben im letzten Jahr von CSR-Massnahmen von Unternehmen gehört. Die wichtigste Informationsquelle ist dabei die Berichterstattung in Medien (93\% der Befragten), gefolgt von Unternehmensbroschüren $(54 \%)$ und der Firmenwerbung (52\%). Die Internetseite eines Unternehmens wird hingegen nur von $17 \%$ der Befragten als Informationsquelle über deren gesellschaftlichen Aktivitäten genannt. Die Bedeutung der Medien als Informationsquelle für Konsumenten in Bezug auf CSR wird durch andere Studien bestätigt (Simon 1992:268ff; Lunau und Wettstein 2004:144). Auch Unternehmen bezeichnen Presseberichte als den wichtigsten Informationskanal für ihr gesellschaftliches Engagement (Bertelsmann 2005:24).

Die geringe Bekanntheit der CSR-Aktivitäten von Unternehmen kann zwei Ursachen haben: entweder ist das CSR-Engagement der Firmen noch relativ bescheiden, oder die Unternehmenskommunikation zu CSR-Themen ist marginal. Tatsächlich machen Unternehmen diesbezügliche Informationen oftmals aus Risikoüberlegungen nicht öffentlich (Pleon Kohtes Klewes 2004:10). Dies steht im Widerspruch zu den Ergebnissen einer weltweiten Studie 
über Stakeholder-Kommunikation: über $40 \%$ der Kunden und NGOs reagieren positiv auf die Berichterstattung über CSR-Aktivitäten (Pleon Kohtes Klewes 2005:65).

\section{Beurteilung von Firmen}

Für eine positive Beurteilung eines Unternehmens bezüglich CSR stehen für die Schweizer Bevölkerung die faire Behandlung von Mitarbeitenden (24\% der Befragten, die ein Unternehmen nennen können) im Vordergrund. Daneben werden, umweltfreundliche Produkte (12\%) und die persönliche Erfahrung (11\%) am häufigsten genannt (siehe Abbildung 3). Die gleichen Themen werden (mit umgekehrten Vorzeichen) auch bei Firmen mit negativer CSR-Beurteilung genannt.

Die Befragten werten die CSR-Performance von Unternehmen des Detailhandels, der Lebensmittel- und der Pharmabranche am positivsten. An der Spitze der Liste der negativ beurteilten Unternehmen finden sich ebenfalls Unternehmen der Lebensmittel- und Pharmabranche neben den Banken (insbesondere Grossbanken) und der Mineralölindustrie. Insgesamt sind die Aussagen zur Bewertung der CSR-Leistung von Firmen insofern zu relativieren, als sie auch Markeneffekte beinhalten und die Bekanntheit von Unternehmen widerspiegeln.

Für den Detailhandel und die Nahrungsmittelindustrie sind bei der Beurteilung insbesondere die Produktions- und Transportbedingungen unter sozialen und ökologischen Gesichtspunkten sowie die transparente und glaubwürdige Deklaration der Produkte wichtig. Banken und Pharmakonzerne können ihre CSR-Performance verbessern, wenn sie eine ausgeglichenere Lohnpolitik verfolgen und nicht nur die kurzfristige Gewinnmaximierung sondern auch längerfristige gesellschaftliche Interessen berücksichtigen. Banken sollten aus Sicht der Schweizer Bevölkerung insbesondere die Anliegen der kleineren Unternehmen und weniger zahlungskräftigen Kunden, die Pharmakonzerne die Bedürfnisse der Entwicklungsländer stärker in ihre CSR-Konzepte miteinbeziehen. Die Erdölbranche kann sich mit Umweltschutz (z.B. Massnahmen zur Minderung des Einflusses der Erdölförderung auf Ökosysteme, Klima) und herausragendem Management von sozialen Themen (z.B. Gesundheitsschutz und Sicherheit) profilieren.

\section{Banken im Fokus}

Beim Swiss CSR Monitor 2006 lag der Schwerpunkt der Befragung auf den Banken und Pensionskassen. Der Finanzsektor nimmt in einer Volkswirtschaft eine zentrale Rolle ein. Er vermittelt zwischen Kapitalangebot und -nachfrage und stellt so den gesamtwirtschaftlichen Geldkreislauf sicher. Aufgrund ihrer wichtigen Rolle sind Banken mit hohen Erwartungen konfrontiert, welche nicht nur ihre Produkte und Dienstleistungen, sondern auch ihre Rolle in der Gesellschaft insgesamt betreffen. Jeder ihrer Funktionen stellen sich im Zusammenhang mit CSR eigene Herausforderungen.

Aus einer vorgegebenen Liste von CSR-Themen in Banken schätzt die Schweizer Bevölkerung die Geldwäschereibekämpfung ( $82 \%$ der Befragten stimmen $\mathrm{zu}$ ) und die faire Behandlung von Mitarbeitenden unabhängig von $\mathrm{Ge}$ schlecht, Abstammung und religiöser Orientierung (78\%) als zentral ein. Nur $46 \%$ der Befragten stimmen hingegen $\mathrm{zu}$, dass Banken Kredit- oder Anlageprodukte aktiv verkaufen sollen, die ökologische, soziale und ethische Aspekte berücksichtigen (sogenannte SRI- oder Socially-Responsible-Investment-Produkte). Ebenso sind nur $47 \%$ der Meinung, dass Banken zur Lösung von weltweiten Problemen wie Bildungsmangel, Menschenrechtsverletzung und Armut beitragen sollen (siehe Abbildung 4).

Ein wichtiger Aspekt von CSR ist das Management der Beziehungen zu den Anspruchsgruppen, denn die Inhalte von CSR ergeben sich aus deren Erwartungen (Carroll 1991). Für die Schweizer steht die Verantwortung der Banken gegenüber den Kundinnen und Kunden (66\% antworten ,voll und ganz verantwortlich“) und den Mitarbeitenden $(62 \%)$ an oberster Stelle. Erst an dritter Stelle werden die Aktionäre genannt $(51 \%)$, wobei Personen, die gemäss eigenen Angaben selbst Aktionäre sind, diese Gruppe wich-
Abb. 4 Verantwortung von Banken

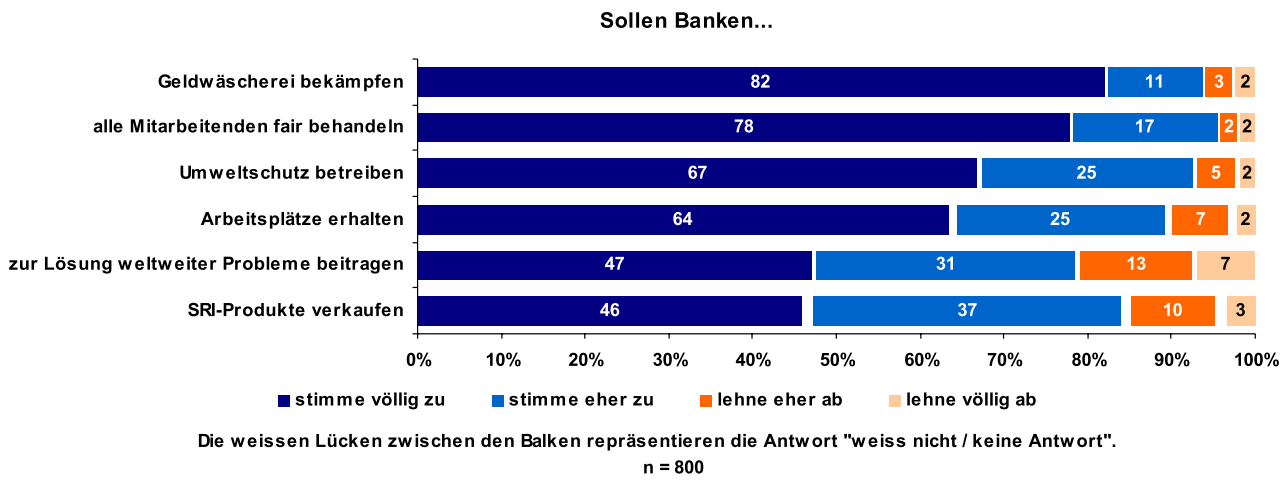

Sollen Banken... 
tiger einschätzen. Interessengruppen wie Umweltverbände oder Menschenrechtsorganisationen werden als die am wenigsten wichtige Anspruchsgruppe eingestuft (18\%). Die Priorisierung der relevanten Stakeholder durch die Befragten entspricht jener der Bankenpraxis (Furrer und Hoffmann 2005). Weder die Banken noch die Schweizer Bevölkerung haben erkannt, welche Rolle Interessengruppen als Einflussfaktoren und Frühindikatoren für sich entwickelnde CSR-Themen spielen können.

\section{Druck auf Pensionskassen}

Auch wenn Versicherte ihre Bedürfnisse bezüglich der Anlagestrategie von Pensionskassen heute noch wenig artikulieren, bestehen klare Vorstellungen. Für 56\% sind sozial verantwortliche Anlagen eine Alternative zu konventionellen Anlagen, wenn sie die Erbringung der Vorsorgeleistung nicht gefährden. Darüber hinaus erwarten $90 \%$ der Befragten von ihrer Vorsorgeeinrichtung eine Massnahme, falls sie in Unternehmen investiert, die soziale und ökologische Standards nicht einhält. Dabei gibt die Schweizer Bevölkerung Massnahmen, die das Investitionsverhalten betreffen (68\% stimmen zu), den Vorzug vor dem Einsatz von Stimmrechten (22\% stimmen zu). Auch zeigt sich ein klares Bedürfnis nach mehr Information. Die Mehrheit der Befragten (80\%) würde es begrüssen, wenn die Pensionskassen gesetzlich dazu verpflichtet würden, sie über allfällige soziale, ökologische und ethische Erwägungen zu informieren, die bei der Auswahl von Anlagen eine Rolle spielen.

\section{Fazit}

Bei vielen grossen Firmen ist in den letzten Jahren eine Sensibilisierung für CSR-Themen festzustellen. Dennoch wird die Glaubwürdigkeit der CSR-Programme von einem Großteil der Befragten in Frage gestellt. Von Unternehmen, welche sich mit CSR im Markt positionieren wollen, ist grössere Transparenz gefordert. Der Dialog mit NGOs kann Unternehmen helfen, frühzeitig branchen- und firmenspezifische Chancen und Risiken im Zusammenhang mit verantwortungsvoller Unternehmensführung zu erkennen und die Glaubwürdigkeit von CSR-Programmen zu erhöhen. Mit dem Anbringen von Hinweisen auf Produkten kann das soziale Verantwortungsbewusstsein wirkungsvoll zum Ausdruck gebracht werden. Neben einer professionellen Medienarbeit kann auch eine herausragende CSR-Berichterstattung zu einem Reputationsaufbau beitragen.
Weitere Informationen und kostenloser Bezug der ca. 70 Seiten umfassenden Studie (als pdf-Datei) bei Bettina Furrer, Institut für Nachhaltige Entwicklung, Zürcher Hochschule Winterthur (www.ine.zwhin.ch; bettina.furrer@zhwin.ch)

\section{Anmerkung}

(1) Das Konzept der „Corporate Social Responsibility“ (CSR) hat eine lange und facettenreiche Geschichte (vgl. dazu Clarkson 1995). Der „Swiss CSR Monitor“ stützt sich auf die Definition von Carroll (1991), der postuliert, dass CSR ein Konzept ist, das alle Geschäftsaktivitäten eines Unternehmens integrieren sollte. Dabei konstituieren vier Typen von unternehmerischer Verantwortung CSR: die ökonomische, rechtliche, ethische und philanthropische Verantwortung.

\section{Literatur}

Bertelsmann Stiftung: Die gesellschaftliche Verantwortung von Unternehmen. Dokumentation der Ergebnisse einer Unternehmensbefragung der Bertelsmann Stiftung, http://www.bertelsmann-stiftung.de/bst/de/media/xcms_bst_dms_16837__2.pdf (11.5.2006). (2005)

Carroll, A. B.: The Pyramid of Corporate Social Responsibility. Business Horizons: 39-48. (1991)

Carroll, A. B.: Corporate Social Responsibility. Evolution of a Definitional Construct. Business \& Society, Vol. 38 38: 268-295. (1999)

Clarkson, M. B. E.: A stakeholder framework for analyzing and evaluating corporate social performance. Academy of Management Review 20(1): 92-117. (1995)

Dawkins, J./S. Lewis: CSR in stakeholder expectations and their implication for strategy. Journal of Business Ethics 44: 185-193. (2003)

Environics International: Corporate Social Responsibility Monitor 2003. Toronto. (2003)

Furrer, B./V. Hoffmann: Identification of Strategic Sustainability Drivers under Uncertainty. International Conference ISC 2005, Basel. Unveröffentlichter Tagungsbeitrag. (2005)

Lunau, Y./F. Wettstein: Die soziale Verantwortung der Wirtschaft. Was Bürger von Unternehmen erwarten. Bern, Haupt. (2004)

Pleon Kohtes Klewes: Geheime Mission? Deutsche Unternehmen im Stakeholder-Dialog. Vergleich Unternehmen allgemein und Finanzdienstleister. Die zweite weltweite Stakeholder-Befragung zur Nachhaltigkeitsberichterstattung von Unternehmen. Bonn, http://www.pleon.de/Studien.1166.0.html (25.5.2006). (2004)

Pleon Kohtes Klewes: Unternehmen Verantwortung: der Global Stakeholder Report 2005. Die zweite weltweite Stakeholder-Befragung zur Nachhaltigkeitsberichterstattung von Unternehmen. Bonn. http://www.pleon.de/fileadmin/downloads/Pleon_GSR05_dt.pdf (11.5.2006). (2005)

Simon, F. L.: Marketing green products in the triad. Columbia Journal of World Business 27 (3,4): 268-285. (1992) 